\title{
diffusion-îuridarrentitals
}

The Open-Access Journal for the Basic Principles of Diffusion Theory, Experiment and Application

\section{Diffusion Exchange NMR Spectroscopic Study of Dextran Exchange through Polyelectrolyte Multilayer Capsules}

\author{
Petrik Galvosas* $^{*}$ Ying Qiao ${ }^{*}$, Paul T. Callaghan ${ }^{*}$, Thorsteinn Adalsteinsson ${ }^{\dagger}$, \\ Monika Schönhoff ${ }^{\dagger}$
}

* Universität Leipzig, Fakultät für Physik und Geowissenschaften, Linnéstr. 5, 04103 Leipzig, Germany, E-Mail: galvosas@physik.uni-leipzig.de

* MacDiarmid Institute for Advanced Materials and Nanotechnology, School of Chemical and Physical Sciences, Victoria University of Wellington, Wellington, New Zealand

† Institut für Physikalische Chemie, Westfälische Wilhelms-Universität Münster, Corrensstr. 30, D-48149 Münster, Germany

\section{Introduction}

Release-tailored encapsulated drugs have a number of advantages over conventional drug forms. For example, they can prolong the time of activity, protect sensitive drugs from human immune system and deliver drug to a specific site in human body, etc. Various microstructures such as liposomes, microgels, microemulsions, polymer micelles and colloids have been widely employed as drug carriers. Recently, a novel microencapsulation technology, based on layer-by-layer assembly of oppositely charged polyelectrolytes [1], onto dissolvable colloidal templates has been established. Unlike liposomes, the polyelectrolyte multilayer (PEM) microcapsules are tough, uniform in size, and with selective and tunable permeability.

This specific permeability of the capsule wall and an exchange between the interior and the exterior of the capsule is of particular interest in the design of release-controlled drugs. In order to directly determine the time scale of the exchange of macromolecules through the capsule wall and the mobility of macromolecules within different domains of the macromolecule/capsule dispersion, we apply diffusion exchange and correlation twodimensional (2D) nuclear magnetic resonance (NMR) techniques to study the diffusion behavior of dextran with a molecular weight of $77 \mathrm{kDa}$, through 4-bilayer thick $(\sim 20 \mathrm{~nm}$, $520 \mathrm{~nm}$ diameter template) polystyrene sulfonate/polydiallyldimethylammonium chloride (PSS/PDADMAC) capsules [2]. Dextran is a water-soluble, bio-polysaccharide that may be formed using enzyme technology, is commercially available and is currently used as drugs. Certain grades of dextran are used as blood plasma volume expanders and irondextran is used to combat iron-deficiency anemia.

\section{Experimental and Results}

Recently developed Pulsed Gradient Spin Echo (PGSE) 2D NMR techniques have significant advantages over their 1D counterparts. Diffusion-Diffusion Exchange Spectroscopy (DEXSY) [3] allows a model-free approach that directly determines quantitative information about exchange processes for a given mixing time $t_{m}$ in a single 2D experiment. Similarly Diffusion-Relaxation Correlation Spectroscopy (DRCOSY) [4] 
may be used to directly correlate diffusion coefficients with $T_{2}$ relaxation times of multiple phases in a single 2D experiment for a given diffusion observation time.

The values of diffusion coefficients found with the DRCOSY are consistent with four states for the dextran: freely diffusing in the bulk, in transient interaction with the capsule surfaces, and confined both within the capsules, and capsule aggregates, albeit with a slow exchange process to the exterior.

Fig. 1 shows the results of a DEXSY measurement obtained at a mixing time of $200 \mathrm{~ms}$. The diagonal peaks correspond to the diffusion of dextran confined in capsules $(10 \%)$ and a joint peak of the free and interacting dextran $(86.8 \%)$. The off-diagonal peaks originate from molecules with a different diffusion coefficient before and after the mixing

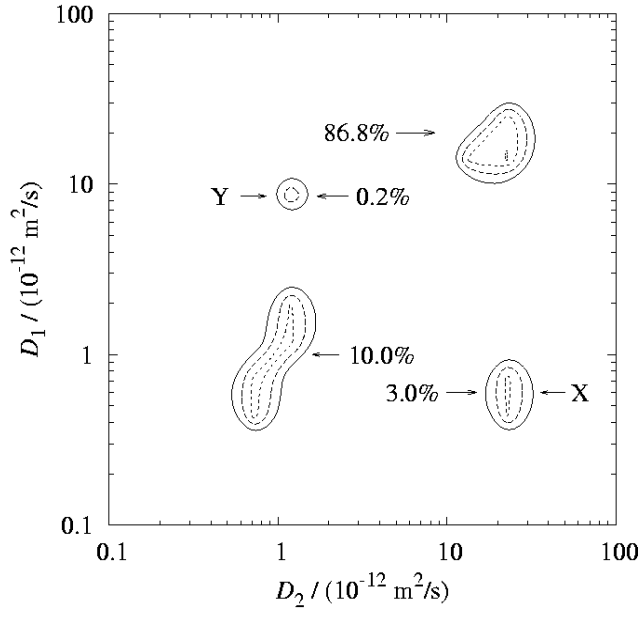

Fig: 1: DEXSY for $t_{m}=200 \mathrm{~ms}$ time, thus undergoing an exchange: Molecules represented by peak " $\mathrm{X}$ " have a small initial diffusion coefficient due to the confinement in the capsules while after the mixing time their diffusion behaviour corresponds to the free dextran. On the contrary, molecules represented by peak "Y" enter the capsules during the mixing time.

\section{Conclusion}

Results obtained in DRCOSY and DEXSY experiments show that a confined phase of dextran molecules is found in the dextran/capsule system. In DRCOSY experiments, the dependence of diffusion coefficients on observation time suggests a restricted diffusion behavior of dextran molecules within the capsules as well as evidence for inter-capsule interactions. We note here that the dextran confined to capsule aggregates dominates the overall dextran signal. Further, we find that dextrans are nearly 30 times more likely to be found in the single capsule and capsule aggregate states compared with an estimate based on volume fraction alone.

About $1.5 \%$ of dextran molecules exchange through capsule walls between the confined phase and the free and interacting phases at $t_{m}=200 \mathrm{~ms}$, suggesting an exchange time on the order of $\sim 1 \mathrm{~s}$. The quantitative information on dextran exchange and diffusion found here prove useful in designing polyelectrolyte multilayer capsules for use as drug carriers.

\section{References}

[1] G. Decher, Science 277 (5330), (1997) 1232

[2] Y. Qiao, P. Galvosas, P. T. Callaghan, T. Adalsteinsson, M. Schönhoff, Journ. Chem. Phys. submitted

[3] P. T. Callaghan and I. Furo, Journ. Chem. Phys. 120 (8), (2004) 4032

[4] S. Godefroy and P. T. Callaghan, Magn. Res. Imag. 21 (3-4), 381 (2003) 